\title{
Structural white-matter connections mediating distinct behavioral components of spatial neglect in right brain-damaged patients
}

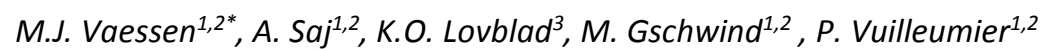

1. Laboratory for Neurology and Imaging of Cognition, Department of Neurosciences, University Medical Centre, Geneva, Switserland

2. Dept of Clinical Neurology, University Hospital of Geneva, Geneva, Switzerland

3. Dept of Radiology, University Hospital of Geneva, Geneva, Switzerland

Correspondence to:

M.J. Vaessen

Campus Biotech

9, Chemin des Mines

Postbox 60

$\mathrm{CH}-1211$ Geneva 20

Switzerland

Email: m.vaessen@gmail.com

Phone: 0041-223790344

Keywords: Clinical neuroanatomy; Imaging; Neglect; Diffusion tensor imaging; Fiber tracking

(C) 2015. This manuscript version is made available under the Elsevier user license http://www.elsevier.com/open-access/userlicense/1.0/ 


\section{ABSTRACT}

Spatial neglect is a neuropsychological syndrome in which patients fail to perceive and orient to stimuli located in the space contralateral to the lesioned hemisphere. It is characterized by a wide heterogeneity in clinical symptoms which can be grouped into distinct behavioral components correlating with different lesion sites. Moreover, damage to white-matter fiber tracts has been suggested to disconnect brain networks that mediate different functions associated with spatial cognition and attention. However, it remains unclear what whitematter pathways are associated with functionally dissociable neglect components. In this study we examined 9 patients with a focal right hemisphere stroke using a series of neuropsychological tests and diffusion tensor imaging (DTI) in order to disentangle the role of specific white-matter pathways in neglect symptoms. First, following previous work, the behavioral test scores of patients were factorized into three independent components reflecting perceptual, exploratory, and object-centred deficits in spatial awareness. We then examined the structural neural substrates of these components by correlating indices of white matter integrity (fractional anisotropy) with the severity of deficits along each profile. Several locations in the right parietal and frontal white matter correlated with neuropsychological scores. Fiber tracts projecting from these locations indicated that posterior parts of the superior longitudinal fasciculus (SLF), as well as nearby callosal fibers connecting ipsilateral and contralateral parietal areas, were associated with perceptual spatial deficits, whereas more anterior parts of SLF and inferior fronto-occipital fasciclus (IFOF) were predominantly associated with object-centred deficits. In addition, connections between frontal areas and superior colliculus were found to be associated with the exploratory deficits. Our results provide novel support to the view that neglect may result from disconnection lesions in distributed brain networks, but also extend these notions by highlighting the role of dissociable circuits in different functional components of the neglect syndrome. However these preliminary findings require replication with larger samples of patients. 


\section{INTRODUCTION}

A common consequence of right hemisphere stroke is spatial hemineglect. Patients with neglect fail to detect (and respond to) stimuli located contralaterally to the lesioned hemisphere (Mesulam, 1999; Vallar \& Perani, 1986), even though basic sensory and motor functions may remain intact. This disorder has been attributed to pathological biases in brain systems controlling the distribution of attention in space, preventing conscious perception of contralesional events and intentional action toward them (Driver \& Vuilleumier, 2001; Heilman \& Van Den Abell, 1980; Mesulam, 1990). Neglect patients often have a poor prognosis for long-term recovery on a wide range of everyday tasks (Jehkonen et al., 2000). A better understanding of this complex syndrome is not only crucial to improve rehabilitation strategies but also key to gain insights into the functioning of normal attention and spatial cognition.

Neglect is characterized by a large heterogeneity, both neuro-anatomically and in terms of clinical manifestations (Vuilleumier, 2013). Patients may show signs of neglect in one test but not in others (Halligan \& Marshall, 1998; Saj, Verdon, Vocat, \& Vuilleumier, 2012; Verdon, Schwartz, Lovblad, Hauert, \& Vuilleumier, 2010). In clinical practice, therefore, spatial neglect is usually diagnosed by using a battery of tests consisting of measures that probe a variety of neglect symptoms (e.g. cancellation, line bisection, drawing, reading, and writing). The idea that spatial neglect is a multi-componential syndrome (Driver, Vuilleumier, \& Husain, 2004) is consistent with brain imaging findings that have related neglect to multiple lesion sites (Mesulam, 1999; Verdon et al., 2010). Whereas it is typically linked to parietal lobe damage (Vallar \& Perani, 1986), neglect deficits also frequently arise after lesions affecting the frontal (Damasio, Damasio, \& Chui, 1980) or temporal lobes (Karnath, Ferber, \& Himmelbach, 2001), as well as thalamus (Damasio et al., 1980; Rafal \& Posner, 1987) or basal ganglia (Karnath, 2002). This anatomical diversity has been further supported by recent meta-analysis suggesting that distinct forms of neglect may be associated with distinct (but also common) lesion sites in both the grey and white matter (Chechlacz, Rotshtein, \& Humphreys, 2012; Molenberghs, Sale, \& Mattingley, 2012). For example, neglect on line bisection tends to correlate with superior parietal damage (Binder, Marshall, Lazar, Benjamin, \& Mohr, 1992; Rorden, Fruhmann Berger, \& Karnath, 2006; Verdon et al., 2010), whereas visual extinction correlates with more inferior parietal lesions (Karnath, Himmelbach, \& Küker, 2003), and neglect on search tasks predominates after frontal or subcortical lesions (Husain \& Kennard, 1996; Verdon et al., 2010).

These neglect symptoms reflect a disruption of brain systems responsible for spatial awareness and attention control, known to rely on distributed networks of several cortical and subcortical areas, as shown by recent research on structural and functional connectivity disturbances after right hemisphere stroke (Bartolomeo, Thiebaut de Schotten, \& Chica, 2012; Corbetta \& Shulman, 2011; He et al., 2007). Among the largescale brain networks highlighted by functional magnetic resonance imaging (fMRI) investigations in healthy people (Smith et al., 2009), two networks are thought to be most relevant for attention and spatial orientation (Bartolomeo et al., 2012; Corbetta \& Shulman, 2011; Fox, Corbetta, Snyder, Vincent, \& Raichle, 2006): the ventral attention network (VAN) and the dorsal attention network (DAN). The bilateral DAN is involved in voluntary top- 
down control, while the right-lateralized VAN is mainly activated by salient (or unexpected) stimuli (Corbetta \& Shulman, 2002). The parietal, temporal, and frontal cortical components of both the VAN and DAN are structurally connected by fiber tracts in the periventricular white matter (WM) (Doricchi, Thiebaut de Schotten, Tomaiuolo, \& Bartolomeo, 2008; Umarova et al., 2010). Converging evidence from studies employing lesion mapping (Karnath, Rorden, \& Ticini, 2009; Thiebaut de Schotten et al., 2012; Verdon et al., 2010) and metaanalyses (Chechlacz, Rotshtein, \& Humphreys, 2012; Molenberghs et al., 2012) point to an important role for disruption of these parieto-frontal and occipito-frontal fiber tracts in right-hemisphere stroke patients with neglect. Recent research has indeed shown that WM tracts thought to inter- and intra-connect these attention networks are frequently damaged in patient with neglect. Notably, Thiebaut de Schotten and colleagues (Thiebaut de Schotten et al., 2012) reported that among two patients with similar patterns of cortical damage, one with neglect had damage to the second branch of the superior longitudinal fasciculus (SLF), which was preserved in the patient without neglect. The same study also showed that disconnection of the arcuate fasciculus (AF) and other sectors of the right SLF was highly predictable of the presence of neglect in a larger group of patients, and its extent correlated with performance on line bisection and letter cancellation tasks. Likewise, (He et al., 2007) observed that the severity of neglect was closely related to subcortical damage overlapping with the position of SLF and AF. However, another study (Urbanski et al., 2011) also highlighted a role for damage to the lower (third) branch of SLF (SLFIII), optic radiations (OR), and inferior fronto-ocipital fasciculus (IFOF). Furthermore, damage to thalamic radiations projecting to/from parietal and frontal cortex might additionally play an important role in spatial attention (Greenberg et al., 2012; Saalmann, Pinsk, Wang, Li, \& Kastner, 2012), although their role in neglect remains unclear (Vuilleumier, 2013). Various lesions encroaching upon the thalamus, basal ganglia, and subcortical white matter near the frontal, temporal, and parietal lobes have been described to produce neglect (He et al., 2007; Karnath, Fruhmann Berger, Küker, \& Rorden, 2004; Ringman, Saver, Woolson, Clarke, \& Adams, 2004), indicating that a disconnection between cortical and subcortical areas can severely disrupt the function of large-scale networks mediating spatial awareness and attention. In addition, damage to inter-hemispheric white-matter connections in the posterior corpus callosum has also been shown to contribute to the severity of neglect (Bozzali et al., 2012), which is consistent with earlier lesion work in monkeys (Gaffan \& Hornak, 1997).

Nonetheless, previous studies of white-matter disconnection in neglect have generally considered patients to either have neglect or not have neglect based on a single test, e.g. see (Vallar \& Perani, 1986) or based on a global score summing performance on several tests, e.g. see (Wilson, Cockburn, \& Halligan, 1987). Although this is a standard practice, patients may thus be diagnosed as having neglect when they fail on, say, tasks $A$ and B (not C) but also when they fail on task C alone and actually show no deficit on other tasks (Vuilleumier, 2013). This approach does not consider the multi-componential nature of neglect and may identify neuroanatomical substrates that are common to a range of tasks, but fail to elucidate more precise functional circuits (Verdon et al., 2010; Vuilleumier, 2013). Moreover, the clinical neglect tasks used for diagnosis are typically designed to be sensitive and detect deficits of different nature, rather than to measure a precise cognitive function or a unique attentional process. For instance, line bisection, cancellation, and drawing are all known to rely on a combination 
of perceptual, motor, and representational abilities (Bisiach, Geminiani, Berti, \& Rusconi, 1990; Di Pellegrino, 1995; Heilman \& Van Den Abell, 1980), but to different degrees - which may account for frequent dissociations in a given patient (Halligan, Marshall, \& Wade, 1989). This notion has been corroborated by neuropsychological studies using factorial analysis which showed that performance across different neglect tasks can be accounted by a few shared underlying factors, reflecting perceptual vs. motor components (Binder et al., 1992; Verdon et al., 2010) or egocentric vs. allocentric components (Chechlacz, Rotshtein, Roberts, et al., 2012; Verdon et al., 2010). Accordingly, a recent lesion mapping study identified three main behavioral components explaining different aspects of spatial neglect (Verdon et al., 2010): (1) a visual-spatial deficit mainly impacting on line bisection and text reading that correlated with posterior parietal lesions, (2) an exploratory deficit mainly affecting selective attention during search that correlated with dorsolateral frontal damage, and (3) an allocentric (object-based) deficit that correlated with temporal lobe damage (Saj et al., 2012; Verdon et al., 2010). However, it remains unclear whether these dissociable components of neglect may also relate to damage to different white-matter tracts participating to different functional networks.

In the current study, we therefore set out to test whether these three distinct components of neglect, shared across different clinical tests, are associated with lesions affecting to specific white-matter regions and fiber tracts. We specifically investigated whether major neglect components (rather than neglect tests) are correlated with distinct patterns of damage to white-matter tracts. We applied a novel strategy based on fiber tracking from seeds defined by lesion mapping of white matter damage in patients with different degrees of deficits along different dimensions of spatial neglect. By using a whole-brain data-driven approach, without restricting analysis to a priori tracts or regions of interest (ROIs), we were able to identify white-matter clusters and fibers distinguishing between patients with good scores vs. those with bad scores on each of the main neglect components.

\section{METHODS}

\section{Patients}

We recruited 9 patients ( 3 males) with a first focal right hemisphere stroke, who were consecutively admitted to the Neurology Department of Geneva University Hospital. The overall distribution of right hemisphere damage among all patients is shown in Fig. 1. No previous brain disease was reported in their medical history. Their mean age was 69 years (range 52-81 years) and the mean time of clinical testing since stroke onset was 12.8 days (range 5-34 days). Neglect was assessed in the acute (one week to three months) stage, when deficits are most pronounced. According to neuropsychological testing (see below), 4 patients showed severe neglect (i.e. deficits on at least 4 of all tests administered) during this phase.

\section{Neuropsychological assessment}


Neglect was assessed in each patient using a systematic battery of standard paper-and-pencil tests that could be easily administered in a clinical setting (Saj et al., 2012; Verdon et al., 2010). Our battery focused on classic tests assessing extra-personal neglect in near space, which have been widely employed in clinical practice and previous studies (Karnath et al., 2004; Mort et al., 2003; Ogden, 1985; Vallar \& Perani, 1986). It included the following tests: Schenk omissions (Schenkenberg, Bradford, \& Ajax, 1980), Bell task omissions (Philippe Azouvi et al., 2003), Mesulam omissions (Weintraub \& Mesulam, 1985), Line bisection (Philippe Azouvi et al., 2003), Text reading (Philippe Azouvi et al., 2003), Clock drawing (Philippe Azouvi et al., 2003), and Scene copying (Gainotti, Messerli, \& Tissot, 1972). Altogether these tests allowed us to probe perceptual, attentional, and visuo-motor activity (P Azouvi et al., 2002), as well as both space-based and object-based processing (Hillis et al., 2005). Complete test scores for all patients can be found in supplementary Table 1.

A factorial analysis was performed on scores of some of these tests from a large right-hemisphere stroke population $(n=80)$ reported in a previous study (Verdon et al., 2010), which included the patients from the current study. This analysis extracted three main factors that explained the most variance in performance across all neglect tests. The loadings of these three factors were then extracted for each patient. More details about the tests and the factorial analysis are provided in Verdon et al. (Verdon et al., 2010).

The first factor regrouped rightward deviation on line bisection and left-sided omissions in word reading (perceptual component). The second factor regrouped the two scores reflecting object-based neglect, i.e. omissions or transformations for the initial left-part of words in the compound-word reading task, plus omissions of targets defined by a gap on their left-side in Ota search task (allocentric component). Finally, the third factor regrouped the number of misses in left space from the two visual cancellation tests (motor-exploratory component) See Table 1 for a listing of the tasks included in each component. The different tests administered to the patients in the current study were categorically assigned to one of the three components according to the factorial analysis of Verdon et al. (2010). For each patient we then computed a score reflecting the severity of impairment on each of these three factors, by summing their z-scores on each of the tests that were included in a particular factor. This categorical assignment of tests to factors allowed a better separation and easier interpretation of differences in the current small sample. Note that a few tests used in the current study differed from those used by Verdon et al. (2010) study (i.e., Mesulam omissions instead of Ota omissions, clock drawing in addition to scene copy), but they probed the same cognitive mechanisms, and different cancellation tests and drawing tests have been shown to be related to the same factor (Verdon et al., 2010). This approach allowed us to verify any generalization of the previous study, while guarding against biases induced by a new factorial analysis in a small sample of patients.

\section{MRI acquisition}


Each patient underwent a standard clinical radiological assessment including MRI and/or CT scans of the brain, according to standard stroke protocols at the Radiology Department of Geneva University Hospital. Brain MRI scans included T1, T2, and fluid attenuated inversion recovery (FLAIR) images, obtained with standard parameters on a 1.5 T Philips Intera scanner.

Diffusion weighted MRI (DWI) images were acquired on average within 6 days after stroke, with a pixel size of $2 \times 2 \mathrm{~mm} 2$, slice thickness $2 \mathrm{~mm}$, and a b-value of $1000 \mathrm{~s} / \mathrm{mm} 2$. An echo planar imaging sequence was used with TE 92 ms, TR 9000 ms, and parallel imaging acceleration factor of 2. A set of 30 gradient directions was used. In addition, a single non-diffusion weighted scan (b0-scan) was obtained. The DWI acquisition time was 5 minutes.

\section{Data analysis}

Spatial processing of the diffusion images (motion and eddy current correction, brain extraction and normalization) were performed with FSL routines (Jenkinson, Beckmann, Behrens, Woolrich, \& Smith, 2012). Diffusion tensors, constrained spherical deconvolution (CSD), FA fits and fiber tractography were performed using routines from the MRtrix 0.2.12 software package (Tournier, Calamante, \& Connelly, 2007). First, individual FA maps were normalized to the MNI space of the template FA map in FSL through the TBSS routines. Because data from contiguous voxels are averaged during normalization with tri-linear interpolation, we ensured minimal deviations from normality and could apply standard GLM analysis. Then, a whole brain general linear model with factor scores, age, and gender as regressors was used to compute the voxel-by-voxel association between FA values and the three factor scores derived from our factorial analysis of neglect symptoms. Lesions were not masked for the analysis and all available brain voxels were included. This analysis resulted in several significant clusters where FA values correlated with factor scores, i.e., severity of clinical deficit along a specific neglect component. Next, for each factor, clusters of significant correlation (as determined by the FSL cluster tool) in the right hemisphere were extracted as ROI seeds for fiber tractography. The cluster tool available in the FSL software package makes use of Gaussian Random Field Theory to infer cluster-wise p-values (Worsley, Evans, Marrett, \& Neelin, 1992).

Note that the time between stroke, neuropsychological testing and MRI acquisition differed between patients (see Table 2). As there might be an effect of testing-delay or MRI-delay on relation with FA values and/or component scores, we tested for any systematic effect but found no correlations between NP-delay and cluster peak FA-values or component scores, nor between MRI-delay and cluster peak FA-values or component scores (Spearman's correlation, all p>0.05).

Probabilistic fiber tracking using the CSD fits was subsequently performed to identify the cortical and subcortical projections of the white-matter ROI's from the previous step. For each participant, a whole brain tractogram was generated with 5,000,000 streamlines in the individual DTI space. This amount of tracks has 
previously been shown to be sufficient to reach a stable and reproducible whole brain tractogram at the individual level (Vaessen et al., 2010; Vaessen et al., 2014). Seeds for the fiber tracking were randomly generated within the WM mask of each individual brain. Fibers traversing the voxels of the GLM ROI's were then selected and converted to a tract density image (TDI) (Calamante, Tournier, Jackson, \& Connelly, 2010) and warped to the standard MNI space using the same transformation as for the FA images in the previous step. The subjects TDI 's were averaged separately for the patients performing best and worst (based on the z-scores computed for each component) on each of the three component scores, allowing us to delineate tracts that were differentially preserved vs damaged as a function of severity of deficits in the different neglect components. Furthermore, a percentage difference map of the two TDI's was calculated (100\% * (TDI_good - TDI_bad) / TDI_bad), which was then visually investigated for regions where fiber projection differed between groups. As an anatomical reference to define regions traversed by the fiber tracks, we made use of the ICBM DTI-81 atlas available from the FSL software package (Oishi et al., 2009), the reference images published in previous work by Forkel and colleagues (Forkel et al., 2014), and those from Catani and colleagues (Catani \& Thiebaut de Schotten, 2008). As mentioned above, there was no correlation between MRI-delay and component scores, and neither was there any difference between the patients with "good" and "bad" neglect scores in term of MRI-delay or NP-test delays values (Independent Samples Median Test, p>0.05).

\section{RESULTS}

\section{Voxel based analysis}

We first computed whole-brain maps identifying clusters of white-matter (WM) where damage (FA values) correlated with the severity of each neglect component. Scatter plots of the cluster FA values versus component scores can be found in the Supplementary Fig. 1.

\section{Perceptual component}

For the visuo-spatial perceptual component (loading on line bisection and text reading), this analysis revealed a large supra-threshold cluster $(p<0.0001)$ predominantly located in the superior corona radiata adjacent to the trunk of the corpus callosum and extending anteriorly below the precentral gyrus (yellow blob, Fig. 2A). A second cluster $(p<0.0001)$ was more posterior and mainly located in the splenium of the corpus callosum (blue blob, Fig. 2A). These cluster involved parts of the brain that were mainly unaffected by macroscopic lesions (see Fig. 1), showing that the DTI analysis could identify areas of damage beyond standard structural mapping of T1 MRI images. 
The exploration component (loading on visual search tasks) revealed clusters mainly located in the frontal lobe. A first cluster (yellow, Fig. 2B, p<0.009) was located in the WM of the superior and middle frontal gyri and adjacent regions of the superior corona radiata. A second cluster (blue, Fig. $2 B, p<0.000$ ) was slightly anterior and inferior to the previous one and included the anterior corona radiata, the WM of the middle frontal gyrus, and parts of the anterior genu of the corpus callosum (forceps minor). Again, these cluster extended in parts of the brain that were mainly unaffected by macroscopic lesions (see Fig. 1).

Figure 1 here

\section{Allocentric component}

For the "object-based" factor (loading on allocentric deficits), three supra-threshold clusters were identified (Fig. $2 \mathrm{C})$. The first $(\mathrm{p}<0.003)$ was situated in the $\mathrm{WM}$ of the superior longitudinal fasciculus and near the supramarginal gyrus (yellow, Fig. $2 \mathrm{C}$ ). The second cluster $(\mathrm{p}<0.002)$ was more anterior within the superior longitudinal fasciculus and extended in the WM of the precentral gyrus (blue, Fig. $2 \mathrm{C})$. The third cluster $(\mathrm{p}<0.000)$ was more posterior and extended more ventrally, overlapping with caudal portions of the superior longitudinal fasciculus but also with the corticospinal tract (green, Fig. 2C).

Altogether, these analyses indicate that the three neglect components correlated with damage to partly different regions of the WM, each predominating in the more posterior, anterior, or intermediate sectors of the SLF and adjacent tracts of the right hemisphere. For this component, only the second cluster was located in a region largely overlapping with the macroscopic lesion.

-Figure 2 here

\section{Fiber tracking}

To identify fiber pathways in the WM that may be interrupted by the lesions identified above, we computed whole-brain maps of fiber tracks (see Methods section) based on the significant clusters that were identified in the previous analysis. Fiber tracking maps were generated using each WM cluster as a seed, from which streamlines were computed to determine projections across WM of the whole brain. To determine differences related to the severity of deficits in each neglect component, we divided the group into patients who performed either in the higher or the lower range (see Table 2 ) on the tests associated with each component (z-scores above or below zero relative to the group mean). We then compared the fiber-tracking maps from each subgroup, by calculating a percentage difference map between streamline density in tract maps from the 'good' and 'bad' patients. Difference maps identified with this procedure revealed WM regions with projections from lesion sites 
associated with specific neglect components, and where tract density was reduced in patients with poor performance on the corresponding neglect tests, relative to patients with good performance. Note that we found no difference maps indicating higher tract density in patients with poor compared to good performance. This data-driven approach allowed us to delineate white-matter connections mediating the different neglect components, without restricting our analysis to pre-defined macroscopic tracts.

\section{Perceptual component}

Using the first cluster associated with this component as a seed, fiber track projections were found to involve parts of the superior longitudinal fasciculus (SLF) in the anterior parietal and posterior temporal regions (see Fig. 3 top row, red), plus parts of the cortico-spinal tract in the anterior capsule, as well as the external capsule beneath the insula (see Fig. 3 bottom row, red), which all exhibited a higher track density in the patients with good test scores on this component, relative to patients with poor scores. Fibers overlapping with the SLF appeared to mainly implicate the second and third branches of this tract (SLF II and III; see Chechlacz, Rotshtein and Humphreys, 2012; Thiebaut de Schotten et al., 2011). Projections from this cluster also involved callosal fibers to the opposite inferior parietal lobule (Fig. 3 middle row, red)

Fiber tracks seeded from the second cluster associated with the perceptual component revealed differences involving similar projections in SLF at the temporo-parietal junction (Fig. 3 top row, blue), but slightly more posterior than projections from the first cluster, as well as bilateral connections passing through the corpus callosum and projecting to posterior parietal areas in both hemispheres (Fig. 3 middle row, blue). Again these differences corresponded to higher tract density for the "good" patients. In addition, smaller and more scattered differences were also seen in posterior WM fibers, projecting toward the occipital cortex (see horizontal section of Fig. 3 bottom row, blue).

Figure 3 here

\section{Exploratory visuo-motor component}

The fiber tracks from the first cluster of this component highlighted projections, with a higher track density for the good patients, in the WM of the superior and middle frontal gyri, near the precentral gyrus (Fig. 4 top row, red). This included WM in the vicinity of the frontal eye fields (see Fig. 4 top and bottom right panels, red), together with a remarkable extension of descending projections toward the upper midbrain in a region corresponding to the superior colliculus (see Fig. 4 bottom row left panels, red).

The second cluster revealed a highly similar pattern extending from the middle frontal gyrus down to the external capsule and upper brainstem, parallel but slightly more anterior than fibers projecting from the 
previous cluster (see Fig. 4 top and bottom right panels, blue). Again, all differences for this component involved stronger track density in good relative to bad patients.

Figure 4 here

\section{Allocentric component}

Results for the third component were globally more widespread. The first cluster for this component showed higher track density in the central fronto-parietal region for the "good" compared to the "bad" patient group (Fig. 5 first and $3^{\text {rd }}$ row, red), projecting to the contralateral hemisphere via the mid-trunk of the corpus callosum (Fig. $52^{\text {nd }}$ row, red) and to the cortico-spinal pathway in the same hemisphere (Fig. $53^{\text {rd }}$ and $4^{\text {th }}$ row, red). Smaller projection differences from this cluster were also observed at the junction between the temporal stem and ventral frontal regions, possibly corresponding to the anterior segment of the inferior fronto-occipital fasciculus (IFOF) (Fig. $51^{\text {st }}, 3^{\text {rd }}$ and $4^{\text {th }}$ row, red).

The second cluster revealed a similar pattern of connections but from slightly more anterior regions in superior frontal gyrus toward subcortical structures (Fig. $51^{\text {st }}, 2^{\text {nd }}$ and $3^{\text {rd }}$ row, blue), as well as connections toward posterior parietal regions overlapping with the superior frontal occipital fasciculus (see Fig. $51^{\text {st }}$ and $2^{\text {nd }}$ row, blue), and toward the mid-trunk of the corpus callosum (Fig. $51^{\text {st }}$ and $2^{\text {nd }}$ row, blue). Again, all projection differences corresponded to higher fiber density in the "good" patient group.

Finally, projections from the third cluster of this component showed some overlap with those from both the first and second clusters above. Differences between good and bad patients were characterized by fiber tracks in the superior frontal gyrus connecting with posterior parietal and subcortical structures (Fig. $54^{\text {th }}$ row, green), including a projection towards the posterior thalamus and pulvinar (see Fig. $54^{\text {th }}$ row, green). Additional tract differences were also found again in the anterior portion of the IFOF and mid-trunk of corpus callosum.

Figure 5 here

\section{DISCUSSION}

In this study we investigated the structural white-matter correlates of different components of spatial neglect, which were previously identified in a large stroke population and shown to explain different kinds of deficits across neglect tests (Verdon et al., 2010). By defining areas in the white matter (WM) associated with more severe deficits in each component and then probing for the fiber track projections passing through these areas, we were able to delineate distinctive patterns of connections associated with each neglect components. Our 
results provide novel support to the notion that different behavioral aspects of neglect are related to spatially distinct sites of white matter injury, which contribute to disconnect functional nodes in large-scale brain networks mediating spatial attention and awareness (Bartolomeo, Thiebaut de Schotten, \& Doricchi, 2007). Our data accord with previous anatomical findings suggesting differential involvement of parietal, frontal, and temporal cortical areas in neglect components related to perceptual, motor-exploratory, and allocentric neglect symptoms, respectively (Marsh \& Hillis, 2008; Verdon et al., 2010), among other possible components (Chechlacz, Rotshtein, \& Humphreys, 2012; Molenberghs et al., 2012); but in addition we also extend these previous findings by pointing to specific white-matter pathways connecting these cortical regions with other brain areas. These data highlight neural circuits subserving distinct cognitive functions associated with different neglect components.

\section{The perceptual component}

This component was previously shown to account for a large degree of variance in performance on neglect tests, mainly associated with spatial biases in line bisection and text reading (Verdon et al., 2010). Here we show that the white matter correlates for this component predominantly involve posterior parietal fiber tracks, with an extension toward the SLF segment near the TPJ (SLF II and III) as well as posterior inter-hemispheric callosal connections (Fig. 3). These DTI data therefore dovetail nicely with previous mapping results (based on T1 MRI scans) that linked this component (Verdon et al., 2010) or line bisection deviation alone (Binder et al., 1992; Rorden et al., 2006) with parietal lobe damage. They also accord well with recent DTI studies reporting an association between spatial neglect and lesions in SLF that may disconnect parietal areas from other brain regions (Thiebaut de Schotten et al., 2012).

A critical role for the inferior parietal lobe in neglect has consistently been reported in many studies (Mesulam, 1999; Vallar, 1998), with a particular focus on cortical areas in the supramarginal (Buxbaum et al., 2004; Doricchi \& Tomaiuolo, 2003) and angular gyrus (Hillis et al., 2005; Mort et al., 2003). The right parietal cortex is thought to be responsible for the representation of spatial locations selected for attention (Fox et al., 2006). Parietal regions near the TPJ are also recruited when shifting attention between locations (Gillebert et al., 2011) and when holding spatial information in working memory (Husain et al., 2001; LaBar, Gitelman, Parrish, \& Mesulam, 1999). By combining multisensory inputs with motor and proprioceptive signals, these regions may have a crucial role in the maintenance of previously explored locations (Husain et al., 2001; Vuilleumier, 2013), e.g. across eye or body movements (Pisella \& Mattingley, 2004; Vuilleumier et al., 2007), a spatial "remapping" process that allows a continuous dynamic updating of location information necessary to build a stable internal representations of space (van Assche, Kebets, Vuilleumier, \& Assal, 2014; Wansard et al., 2014). Such a function is likely to be important for locating and maintaining the end-point of a line during bisection or the beginning of the next line in a text during reading, two abilities associated with deficits in our first neglect component (Verdon et al., 2010; Vuilleumier, 2013). Disconnecting WM fibers between posterior parietal areas and more anterior areas in the frontal cortex (possibly conveying motor signals about eye and body movements; see Merriam et 
al., 2003) or the posterior temporal cortex (possibly conveying vestibular signals; see (Andersen, Shenoy, Snyder, Bradley, \& Crowell, 1999; zu Eulenburg, Caspers, Roski, \& Eickhoff, 2012) might critically contribute to deficits in spatial remapping and many perceptual-spatial deficits in neglect. Disconnection by lesion of the SLF might also interrupt functional interactions between the dorsal and ventral attention networks (He et al., 2007) or between the parietal and frontal nodes of these networks (Karnath et al., 2009; Thiebaut de Schotten et al., 2012).

Furthermore, we found that deficits in this perceptual-spatial component of neglect were also associated with damage to posterior inter-hemispheric tracts passing through the splenium of the CC (Fig. 3). This adds to a recent DTI study using tract-based spatial statistics (TBSS) of DTI data in right stroke patients (Bozzali et al., 2012), which reported that micro-structural changes in WM of the posterior CC (lower FA values) predicted more severe neglect of a battery of neglect tasks. WM tracts in the splenium allows for the interhemispheric transfer of visual information and may play an important role in modulating hemispheric rivalry during attention orienting (Bozzali et al., 2012; Kinsbourne, 1977; Urbanski et al., 2008). According to classic models of neglect (Kinsbourne, 1977), parieto-frontal circuits in each hemisphere control the distribution of attention to contralateral space and inhibit orienting in the opposite direction through reciprocal inhibition of the other hemisphere. Damage to the dominant right-hemisphere would reduce inhibition of the left hemisphere and thus result in increased attention toward the ipsilesional/right visual field, leading to the typical perceptual biases observed in neglect patients (Koch et al., 2008; Vuilleumier, Hester, Assal, \& Regli, 1996), including those seen during line bisection and reading. Damage to callosal fibers might directly contribute to such loss of interhemispheric balance in spatial attention. Alternatively, or additionally, an interruption of interhemispheric visual transfer might also disrupt spatial updating processes when visual information has to be remapped from the intact to the damaged hemisphere across eye or body movements (Merriam et al., 2003; Pisella \& Mattingley, 2004; Vuilleumier et al., 2007). Interestingly, lesion studies in the monkey (Gaffan \& Hornak, 1997) have shown that long-lasting neglect symptoms are observed only when WM lesions in parietal lobe are combined with a section of the posterior corpus callosum depriving parietal areas from visual information from both visual fields, and thus precluding the generation of a coherent visual representation of the environment. Such interhemispheric disconnection might therefore play an important role in the perceptual-spatial component of neglect, leading to particular deficits in processing the left-sided line endpoints during bisection or reading.

\section{The exploratory visuo-motor component}

This neglect component primarily reflected target omission in left space during cancellation tests (Verdon et al., 2010). This component has consistently been associated with frontal areas across several studies and metaanalyses (Binder et al., 1992; Bisiach et al., 1990), although a contribution of posterior parietal areas is also likely during visual search tasks (Chechlacz, Rotshtein, \& Humphreys, 2012; Donner et al., 2000; Gillebert et al., 2011; Verdon et al., 2010). This anatomical association appears consistent with a crucial role of the dorsolateral prefrontal areas in the control of attention through the implementation of top-down selectivity signals and inhibition of irrelevant information (Capotosto, Babiloni, Romani, \& Corbetta, 2009; Peers et al., 2005), which 
are both at play during visual search (Husain \& Kennard, 1997; Purcell, Heitz, Cohen, \& Schall, 2012). Here, we found that this exploratory-motor component was associated with distinctive patterns of WM damage predominating in the frontal lobe, in close vicinity of the middle frontal gyrus where the frontal eye field (FEF) is typically located (Amiez \& Petrides, 2009) (see Fig. 4). The latter region is critically implicated in top-down selection of visual targets (Noudoost, Chang, Steinmetz, \& Moore, 2010). Strikingly, our fiber tracking data further revealed that these connections projected to an extended network not only involving posterior WM regions in the parietal lobe, in agreement with previous lesion mapping and DTI studies (Corbetta \& Shulman, 2002; Mesulam, 1999; Thiebaut de Schotten et al., 2012), but also encompassing subcortical structures in the upper brainstem that corresponded to the ipsilateral superior colliculus (see Fig. 4). Very similar projection sites were found when tracking fibers from the two lesion clusters associated with this neglect component, providing some support for the robustness of this finding. These descending connections appear consistent with the existence of direct projections from FEF to subcortical structures including superior colliculus (Schiller, True, \& Conway, 1980), as well as basal ganglia (Neggers et al., 2012) and medial thalamus (Behrens et al., 2003), which are implicated in reflexive oculo-motor control and attention (Corbetta, 1998; Schiller et al., 1980). We cautionary note that the existence of a direct connection between the FEF and the superior colliculus has not been unambiguously shown in the human brain. However, there is ample evidence for the existence of this connection in the animal brain (Borra, Gerbella, Rozzi, \& Luppino, 2013; Cerkevich, Lyon, Balaram, \& Kaas, 2014; Komatsu \& Suzuki, 1985; Meredith, 1999), and it is likely to play a role in attention and neglect (Ogourtsova, Korner-Bitensky, \& Ptito, 2010).

It has previously been suggested that direct damage to FEF might contribute to spatial biases in attention and exploration during search (Bartolomeo et al., 2012; Vuilleumier et al., 1996), particularly in the presence of distractors (Husain \& Kennard, 1997). Our new results indicate that an interruption of its direct connections to the superior colliculus (Schiller et al., 1980) might play an important role in such biases, possibly through a disruption of orienting toward the contralesional side and/or disinhibition of reflexive orienting towards the ipsilesional side subsequent to release of the opposite superior colliculus activity (Lomber, Malhotra, \& Sprague, 2007; Sprague, 1966; Vuilleumier et al., 1996). Although still largely ignored and underexplored (Ogourtsova et al., 2010), an imbalance in superior colliculus circuits mediating spatial orienting and selective attention has previously been suggested to contribute to spatial neglect in several studies with animal models (Lomber et al., 2007; Sprague, 1966; Valero-Cabré, Payne, \& Pascual-Leone, 2007; Valero-Cabré, Payne, Rushmore, Lomber, \& Pascual-Leone, 2005), as well as indirect evidence from psychophysical studies of reflexive attention in humans (Anderson \& Rees, 2011; Bourgeois, Chica, Migliaccio, Thiebaut de Schotten, \& Bartolomeo, 2012; Rafal, Posner, Friedman, Inhoff, \& Bernstein, 1988; Sapir, Soroker, Berger, \& Henik, 1999). A dysfunction in the subcortical collicular pathways could impair visual-sensory-motor integration during attention orienting and thus exacerbate the dysfunction in preserved parts of the fronto-parietal attention networks (He et al., 2007; Kleinschmidt \& Vuilleumier, 2013; Vuilleumier, 2013), contributing to interhemispheric imbalance and spatial neglect. A crucial involvement of these circuits during visual search tasks would be highly consistent with the important demands on both exploratory-motor processes and inhibition of irrelevant distractor information 
during search (Mannan et al., 2005). Future research should further disentangle the role of these two processes in the exploratory-motor component of neglect and their correlates in frontal-colliculus projections.

\section{The allocentric component}

This behavioral component reflects neglect symptoms expressed in object-centered (allocentric) coordinates, characterized by misses for visual information located on the relative left side of shapes or words, irrespective of their absolute location in egocentric space. Several lesion mapping studies have linked this component with damage to the temporal lobe (Chechlacz, Rotshtein, \& Humphreys, 2012; Hillis et al., 2005; Karnath et al., 2001; Karnath et al., 2004; Ringman et al., 2004; Rorden et al., 2006; Verdon et al., 2010), although there is also evidence that allocentric and egocentric neglect may often occur together and share partly common neural substrates (Li, Karnath, \& Rorden, 2014). However, the current results did not show a clear involvement of the temporal lobe. This finding indicates that lesion mapping based on white-matter fibers and more standard analysis of gray matter may provide distinct, but complementary information. Moreover, the WM connection patterns distinguishing patients with good relative to bad performance in these tests appeared more widespread and scattered than the fiber tracts identified for the other two components. WM fibers originating from the superior frontal and posterior parietal lobe were the more prominent, together with an involvement of the anterior part of IFOF (from two clusters), and variable extensions to the mid corpus callosum and to subcortical (mainly posterior thalamic) regions (see Fig. 5). Notably, both lateral and medial temporal areas are known to have strong reciprocal connections with the posterior parietal cortex (Catani, Howard, Pajevic, \& Jones, 2002), and both are critically involved in processing visual object shape and words (Vuilleumier et al., 2007). The IFOF also allows for direct and bi-directional connections between visual areas in the temporo-occipital cortex and inferior frontal areas implicated in object categorization and working memory (Bar, 2003; Forkel et al., 2014). An involvement of IFOF in neglect was also suggested by a recent DTI mapping study in stroke patients (Urbanski et al., 2008). However, this disconnection was not correlated with the severity of neglect on visual search or line bisection tasks (Thiebaut de Schotten et al., 2012; Urbanski et al., 2011), indirectly suggesting that IFOF lesions may relate to other neglect symptoms. The WM connections identified in our study (in both superior and inferior long associative tracts) might therefore constitute direct pathways through which attentional processes controlled by frontal and parietal cortices operate on object-based representations stored in temporal and occipital cortices (Caramazza \& Hillis, 1990; Chechlacz, Rotshtein, Roberts, et al., 2012). Alternatively, these pathways might mediate the influence of egocentric spatial biases in object-based tasks and account for the interaction between allocentric and egocentric coding during these tasks (Driver \& Pouget, 2000; Rorden et al., 2012).

Finally, we note that the object-based component of neglect was also associated with differential involvement of projections to the posterior thalamus (see Fig. 5) in a region overlapping with the pulvinar nucleus. The latter structure has been involved in neglect symptoms (Karnath, 2002), but also in the coordination of attention-related activity in distant cortical areas (Saalmann et al., 2012; Shipp, 2004). Lesions disconnecting 
the pulvinar from frontal and parietal areas might disrupt the integration between egocentric processes (dependent on parietal and/or frontal areas) and allocentric processes (Driver \& Pouget, 2000; Rorden et al., 2012), leading to object-based biases in spatial tasks.

However, given the relatively small sample size of our study, and the fact that only 3 out of 9 patients showed low performance on this object-based component (compared to 5 out of 9 for the other two components, see Table 1), it remains possible that the lack of results in the temporal lobe could reflect insufficient variance in this component score, precluding the detection of reliable effects in the temporal lobe. Future studies with larger number of patients might resolve this issue and should further explore the role of fronto-occipital and pulvinar connections in allocentric and object-based attentional processing.

\section{Methodological considerations}

A note of caution concerns the limited sample size of our study. A larger patient population might have enabled us to use a more robust statistical approach to evaluate differences in tract density between the defined patient groups taking in to account the statistical nature of track count indices and multiple comparison correction, although such methods are still undergoing active development and are not straightforward (Calamante, Smith, Tournier, Raffelt, \& Connelly, 2015). Here we relied on a simple contrast between groups and visual evaluation of fiber projections, which remains descriptive but allowed us to uncover several novel findings that might have been lost with other, more stringent statistical approaches. Nevertheless, our findings show the validity and usefulness of our approach based on dissociable components and tractograms from white-mater ROls.

\section{Conclusions}

Our study shows that the multi-component dimensions of neglect are reflected in distinct patterns of whitematter damage with specific correlates for different aspects of spatial neglect symptoms. Our results provide novel support to the view that neglect may result from disconnection lesions in WM and reflect dysfunction of distributed brain networks, but in addition also extend these notions by highlighting the role of dissociable circuits in different functional components of the neglect syndrome. In sum, our data suggest that WM damage in PPC might contribute to the perceptual-scanning component of neglect by interrupting both interhemispheric and intrahemispheric SLF, perhaps due to the unique position of parietal WM at the crossroad of these two pathways; whereas the exploratory-motor component might contribute to the motor-exploratory component of neglect by interrupting cortico-subcortical connections between FEF and superior colliculus as well as cortico-cortical connections between frontal and parietal areas. Consistent with previous studies (Hillis et al., 2005; Rorden et al., 2012; Verdon et al., 2010), the object-based/allocentric component appears more complex to delineate but likely to involve a network connecting frontal with both temporal and occipital areas mediating the distribution of spatial attention within (rather than between) visual objects. These findings agree with, but go beyond, previous lesion mapping work in neglect, while they also globally accord with but provide new 
constraints on theoretical models of spatial attention and orientation. Future research should more thoroughly explore the role of these distinct WM pathways in specific and well defined cognitive processes, in larger sample of stroke patients with or without clinical neglect, as well as in healthy brains. 


\section{Acknowledgements}

This work was supported by a Marie Curie fellowship to MJV and received funding from the European Union Seventh Framework Programme (FP7/2007-2013) under grant agreement $n^{\circ} 267171$, an SNF grant to PV (SNF 3200B0-114014, and a grant from the University Hospital Geneva to MJV (PRD 10-2013-II). PV was also supported by an award from the Geneva Academic Society (Foremane Fund). 


\section{REFERENCES}

Amiez, C., \& Petrides, M. (2009). Anatomical organization of the eye fields in the human and non-human primate frontal cortex. Progress in Neurobiology, 89(2), 220-30.

Andersen, R. A., Shenoy, K. V, Snyder, L. H., Bradley, D. C., \& Crowell, J. A. (1999). The contributions of vestibular signals to the representations of space in the posterior parietal cortex. Annals of the New York Academy of Sciences, 871, 282-92.

Anderson, E. J., \& Rees, G. (2011). Neural correlates of spatial orienting in the human superior colliculus. Journal of Neurophysiology, 106(5), 2273-84.

Azouvi, P., Olivier, S., de Montety, G., Samuel, C., Louis-Dreyfus, A., \& Tesio, L. (2003). Behavioral assessment of unilateral neglect: study of the psychometric properties of the Catherine Bergego Scale. Archives of Physical Medicine and Rehabilitation, 84(1), 51-7.

Azouvi, P., Samuel, C., Louis-Dreyfus, A., Bernati, T., Bartolomeo, P., Beis, J.-M., ... Rousseaux, M. (2002). Sensitivity of clinical and behavioural tests of spatial neglect after right hemisphere stroke. Journal of Neurology, Neurosurgery, and Psychiatry, 73(2), 160-6.

Bar, M. (2003). A cortical mechanism for triggering top-down facilitation in visual object recognition. Journal of Cognitive Neuroscience, 15(4), 600-9.

Bartolomeo, P., Thiebaut de Schotten, M., \& Chica, A. B. (2012). Brain networks of visuospatial attention and their disruption in visual neglect. Frontiers in Human Neuroscience, 6(May), 110.

Bartolomeo, P., Thiebaut de Schotten, M., \& Doricchi, F. (2007). Left unilateral neglect as a disconnection syndrome. Cerebral Cortex (New York, N.Y. : 1991), 17(11), 2479-90.

Behrens, T. E. J., Johansen-Berg, H., Woolrich, M. W., Smith, S. M., Wheeler-Kingshott, C. a M., Boulby, P. a, ... Matthews, P. M. (2003). Non-invasive mapping of connections between human thalamus and cortex using diffusion imaging. Nature Neuroscience, 6(7), 750-7.

Binder, J., Marshall, R., Lazar, R., Benjamin, J., \& Mohr, J. P. (1992). Distinct syndromes of hemineglect. Archives of Neurology, 49(11), 1187-94.

Bisiach, E., Geminiani, G., Berti, A., \& Rusconi, M. L. (1990). Perceptual and premotor factors of unilateral neglect. Neurology, 40(8), 1278-81.

Borra, E., Gerbella, M., Rozzi, S., \& Luppino, G. (2013). Projections from Caudal Ventrolateral Prefrontal Areas to Brainstem Preoculomotor Structures and to Basal Ganglia and Cerebellar Oculomotor Loops in the Macaque. Cerebral Cortex (New York, N.Y. : 1991), (March), 748-764.

Bourgeois, A., Chica, A. B., Migliaccio, R., Thiebaut de Schotten, M., \& Bartolomeo, P. (2012). Cortical control of inhibition of return: evidence from patients with inferior parietal damage and visual neglect.

Neuropsychologia, 50(5), 800-9.

Bozzali, M., Mastropasqua, C., Cercignani, M., Giulietti, G., Bonnì, S., Caltagirone, C., \& Koch, G. (2012). Microstructural damage of the posterior corpus callosum contributes to the clinical severity of neglect. PloS One, 7(10), e48079.

Buxbaum, L. J., Ferraro, M. K., Veramonti, T., Farne, A., Whyte, J., Ladavas, E., ... Coslett, H. B. (2004). Hemispatial neglect: Subtypes, neuroanatomy, and disability. Neurology, 62(5), 749-56.

Calamante, F., Smith, R. E., Tournier, J.-D., Raffelt, D., \& Connelly, A. (2015). Quantification of voxel-wise total fibre density: Investigating the problems associated with track-count mapping. Neurolmage, 117, $284-93$.

Calamante, F., Tournier, J.-D., Jackson, G. D., \& Connelly, A. (2010). Track-density imaging (TDI): superresolution white matter imaging using whole-brain track-density mapping. Neurolmage, 53(4), 1233-43.

Capotosto, P., Babiloni, C., Romani, G. L., \& Corbetta, M. (2009). Frontoparietal cortex controls spatial attention through modulation of anticipatory alpha rhythms. The Journal of Neuroscience : The Official Journal of the Society for Neuroscience, 29(18), 5863-72. 
Caramazza, A., \& Hillis, A. E. (1990). Spatial representation of words in the brain implied by studies of a unilateral neglect patient. Nature, 346(6281), 267-9.

Catani, M., Howard, R. J., Pajevic, S., \& Jones, D. K. (2002). Virtual in Vivo Interactive Dissection of White Matter Fasciculi in the Human Brain. Neurolmage, 17(1), 77-94.

Catani, M., \& Thiebaut de Schotten, M. (2008). A diffusion tensor imaging tractography atlas for virtual in vivo dissections. Cortex; a Journal Devoted to the Study of the Nervous System and Behavior, 44(8), 1105-32.

Cerkevich, C. M., Lyon, D. C., Balaram, P., \& Kaas, J. H. (2014). Distribution of cortical neurons projecting to the superior colliculus in macaque monkeys. Eye and Brain, 2014(6), 121-137.

Chechlacz, M., Rotshtein, P., \& Humphreys, G. W. (2012). Neuroanatomical Dissections of Unilateral Visual Neglect Symptoms: ALE Meta-Analysis of Lesion-Symptom Mapping. Frontiers in Human Neuroscience, 6(August), 230.

Chechlacz, M., Rotshtein, P., Roberts, K. L., Bickerton, W.-L., Lau, J. K. L., \& Humphreys, G. W. (2012). The prognosis of allocentric and egocentric neglect: evidence from clinical scans. PloS One, 7(11), e47821.

Corbetta, M. (1998). Frontoparietal cortical networks for directing attention and the eye to visual locations: Identical, independent, or overlapping neural systems? Proceedings of the National Academy of Sciences, 95(3), 831-838.

Corbetta, M., \& Shulman, G. L. (2002). Control of goal-directed and stimulus-driven attention in the brain. Nature Reviews. Neuroscience, 3(3), 201-15.

Corbetta, M., \& Shulman, G. L. (2011). Spatial neglect and attention networks. Annual review of neuroscience (Vol. 34).

Damasio, A. R., Damasio, H., \& Chui, H. C. (1980). Neglect following damage to frontal lobe or basal ganglia. Neuropsychologia, 18(2), 123-132.

Di Pellegrino, G. (1995). Clock-drawing in a case of left visuo-spatial neglect: A deficit of disengagement? Neuropsychologia, 33(3), 353-358.

Donner, T., Kettermann, A., Diesch, E., Ostendorf, F., Villringer, A., \& Brandt, S. A. (2000). Involvement of the human frontal eye field and multiple parietal areas in covert visual selection during conjunction search. The European Journal of Neuroscience, 12(9), 3407-14.

Doricchi, F., Thiebaut de Schotten, M., Tomaiuolo, F., \& Bartolomeo, P. (2008). White matter (dis)connections and gray matter (dys)functions in visual neglect: gaining insights into the brain networks of spatial awareness. Cortex; a Journal Devoted to the Study of the Nervous System and Behavior, 44(8), 983-95.

Doricchi, F., \& Tomaiuolo, F. (2003). The anatomy of neglect without hemianopia: a key role for parietal-frontal disconnection? Neuroreport, 14(17), 2239-43.

Driver, J., \& Pouget, A. (2000). Object-Centered Visual Neglect, or Relative Egocentric Neglect? Journal of Cognitive Neuroscience, 12(3), 542-545.

Driver, J., \& Vuilleumier, P. (2001). Perceptual awareness and its loss in unilateral neglect and extinction. Cognition, 79(1), 39-88.

Driver, J., Vuilleumier, P., \& Husain, M. (2004). Spatial Neglect and Extinction. M I T PRESS.

Forkel, S. J., Thiebaut de Schotten, M., Kawadler, J. M., Dell'Acqua, F., Danek, A., \& Catani, M. (2014). The anatomy of fronto-occipital connections from early blunt dissections to contemporary tractography. Cortex; a Journal Devoted to the Study of the Nervous System and Behavior, 56(iii), 73-84.

Fox, M. D. M., Corbetta, M., Snyder, A. Z., Vincent, J. L., \& Raichle, M. E. (2006). Spontaneous neuronal activity distinguishes human dorsal and ventral attention systems. Proceedings of the National Academy of Sciences of the United States of America, 103(26), 10046-51.

Gaffan, D., \& Hornak, J. (1997). Visual neglect in the monkey. Representation and disconnection. Brain : A Journal of Neurology, 120 ( Pt 9, 1647-57. 
Gainotti, G., Messerli, P., \& Tissot, R. (1972). Qualitative analysis of unilateral spatial neglect in relation to laterality of cerebral lesions. Journal of Neurology, Neurosurgery, and Psychiatry, 35(4), 545-50.

Gillebert, C. R., Mantini, D., Thijs, V., Sunaert, S., Dupont, P., \& Vandenberghe, R. (2011). Lesion evidence for the critical role of the intraparietal sulcus in spatial attention. Brain : A Journal of Neurology, 134(Pt 6), 1694-709.

Greenberg, A. S., Verstynen, T., Chiu, Y.-C., Yantis, S., Schneider, W., \& Behrmann, M. (2012). Visuotopic cortical connectivity underlying attention revealed with white-matter tractography. The Journal of Neuroscience : The Official Journal of the Society for Neuroscience, 32(8), 2773-82.

Halligan, P. W., \& Marshall, J. C. (1998). Visuospatial neglect: the ultimate deconstruction? Brain and Cognition, $37(3), 419-38$.

Halligan, P. W., Marshall, J. C., \& Wade, D. T. (1989). Visuospatial neglect: underlying factors and test sensitivity. Lancet, 2(8668), 908-11.

He, B. J., Shulman, G. L., Snyder, A. Z., \& Corbetta, M. (2007). The role of impaired neuronal communication in neurological disorders. Current Opinion in Neurology, 20(6), 655-60.

He, B. J., Snyder, A. A. Z., Vincent, J. J. L., Epstein, A., Gordon, L., \& Shulman, G. L. (2007). Breakdown of functional connectivity in frontoparietal networks underlies behavioral deficits in spatial neglect. Neuron, 53, 905-918.

Heilman, K. M., \& Van Den Abell, T. (1980). Right hemisphere dominance for attention The mechanism underlying hemispheric asymmetries of inattention (neglect). Neurology, 30(3), 327.

Hillis, A. E., Newhart, M., Heidler, J., Barker, P. B., Herskovits, E. H., \& Degaonkar, M. (2005). Anatomy of spatial attention: insights from perfusion imaging and hemispatial neglect in acute stroke. The Journal of Neuroscience : The Official Journal of the Society for Neuroscience, 25(12), 3161-7.

Husain, M., \& Kennard, C. (1996). Visual neglect associated with frontal lobe infarction. Journal of Neurology, 243(9), 652-657.

Husain, M., \& Kennard, C. (1997). Distractor-dependent frontal neglect. Neuropsychologia, 35(6), 829-41.

Husain, M., Mannan, S., Hodgson, T., Wojciulik, E., Driver, J., \& Kennard, C. (2001). Impaired spatial working memory across saccades contributes to abnormal search in parietal neglect. Brain : A Journal of Neurology, 124(Pt 5), 941-52.

Jehkonen, M., Ahonen, J. P., Dastidar, P., Koivisto, A. M., Laippala, P., Vilkki, J., \& Molnár, G. (2000). Visual neglect as a predictor of functional outcome one year after stroke. Acta Neurologica Scandinavica, 101(3), 195-201.

Jenkinson, M., Beckmann, C. F., Behrens, T. E. J., Woolrich, M. W., \& Smith, S. M. (2012). FSL. Neurolmage, 62(2), 782-90.

Karnath, H., Himmelbach, M., \& Küker, W. (2003). The cortical substrate of visual extinction. Neuroreport.

Karnath, H. O., Ferber, S., \& Himmelbach, M. (2001). Spatial awareness is a function of the temporal not the posterior parietal lobe. Nature, 411(6840), 950-3.

Karnath, H.-O. (2002). The subcortical anatomy of human spatial neglect: putamen, caudate nucleus and pulvinar. Brain, 125(2), 350-360.

Karnath, H.-O., Fruhmann Berger, M., Küker, W., \& Rorden, C. (2004). The anatomy of spatial neglect based on voxelwise statistical analysis: a study of 140 patients. Cerebral Cortex (New York, N.Y. : 1991), 14(10), 1164-72.

Karnath, H.-O., Rorden, C., \& Ticini, L. F. (2009). Damage to white matter fiber tracts in acute spatial neglect. Cerebral Cortex (New York, N.Y. : 1991), 19(10), 2331-7.

Kinsbourne, M. (1977). Hemi-neglect and hemisphere rivalry. Advances in Neurology, 18, 41-9.

Kleinschmidt, A., \& Vuilleumier, P. (2013). Disconnecting cognition. Current Opinion in Neurology, 26(4), 333-8. 
Koch, G., Oliveri, M., Cheeran, B., Ruge, D., Lo Gerfo, E., Salerno, S., ... Caltagirone, C. (2008). Hyperexcitability of parietal-motor functional connections in the intact left-hemisphere of patients with neglect. Brain : $A$ Journal of Neurology, 131(Pt 12), 3147-55.

Komatsu, H., \& Suzuki, H. (1985). Projections from the functional subdivisions of the frontal eye field to the superior colliculus in the monkey. Brain Research, 327(1-2), 324-327.

LaBar, K. S., Gitelman, D. R., Parrish, T. B., \& Mesulam, M. (1999). Neuroanatomic overlap of working memory and spatial attention networks: a functional MRI comparison within subjects. Neurolmage, 10(6), 695704.

Li, D., Karnath, H.-O., \& Rorden, C. (2014). Egocentric representations of space co-exist with allocentric representations: evidence from spatial neglect. Cortex; a Journal Devoted to the Study of the Nervous System and Behavior, 58, 161-9.

Lomber, S. G., Malhotra, S., \& Sprague, J. M. (2007). Restoration of acoustic orienting into a cortically deaf hemifield by reversible deactivation of the contralesional superior colliculus: the acoustic "Sprague Effect". Journal of Neurophysiology, 97(2), 979-93.

Mannan, S. K., Mort, D. J., Hodgson, T. L., Driver, J., Kennard, C., \& Husain, M. (2005). Revisiting previously searched locations in visual neglect: role of right parietal and frontal lesions in misjudging old locations as new. Journal of Cognitive Neuroscience, 17(2), 340-54.

Marsh, E. B., \& Hillis, A. E. (2008). Dissociation between egocentric and allocentric visuospatial and tactile neglect in acute stroke. Cortex; a Journal Devoted to the Study of the Nervous System and Behavior, 44(9), 1215-20.

Meredith, M. A. (1999). The frontal eye fields target multisensory neurons in cat superior colliculus. Experimental Brain Research, 128(4), 460-470.

Merriam, E. P., Genovese, C. R., \& Colby, C. L. (2003). Spatial updating in human parietal cortex. Neuron, 39(2), 361-73.

Mesulam, M. (1990). Large-scale neurocognitive networks and distributed processing for attention, language, and memory. Annals of Neurology, 28(5), 597-613.

Mesulam, M. M. (1999). Spatial attention and neglect: parietal, frontal and cingulate contributions to the mental representation and attentional targeting of salient extrapersonal events. Philosophical Transactions of the Royal Society of London. Series B, Biological Sciences, 354(1387), 1325-46.

Molenberghs, P., Sale, M. V, \& Mattingley, J. B. (2012). Is there a critical lesion site for unilateral spatial neglect? A meta-analysis using activation likelihood estimation. Frontiers in Human Neuroscience, 6(April), 78.

Mort, D. J., Malhotra, P., Mannan, S. K., Rorden, C., Pambakian, A., Kennard, C., \& Husain, M. (2003). The anatomy of visual neglect. Brain : A Journal of Neurology, 126(Pt 9), 1986-97.

Neggers, S. F. W., Diepen, R. M. van, Zandbelt, B. B., Vink, M., Mandl, R. C. W., \& Gutteling, T. P. (2012). A functional and structural investigation of the human fronto-basal volitional saccade network. PloS One, 7(1), e29517.

Noudoost, B., Chang, M. H., Steinmetz, N. A., \& Moore, T. (2010). Top-down control of visual attention. Current Opinion in Neurobiology, 20(2), 183-90.

Ogden, J. A. (1985). Anterior-posterior interhemispheric differences in the loci of lesions producing visual hemineglect. Brain and Cognition, 4(1), 59-75.

Ogourtsova, T., Korner-Bitensky, N., \& Ptito, A. (2010). Contribution of the superior colliculi to post-stroke unilateral spatial neglect and recovery. Neuropsychologia, 48(9), 2407-16.

Oishi, K., Faria, A., Jiang, H., Li, X., Akhter, K., Zhang, J., Hsu, J., Miller, M., van Zijl, P., Albert, M, Lyketsos, C., Woods, R, Toga, A., Pike, G., Rosa-Neto, P, Evans, A, Mazziotta, J, Mori, S. (2009). Atlas-based whole brain white matter analysis using large deformation diffeomorphic metric mapping: application to normal elderly and Alzheimer's disease participants. Neurolmage, 46(2), 486-99. 
Peers, P. V, Ludwig, C. J. H., Rorden, C., Cusack, R., Bonfiglioli, C., Bundesen, C., Driver, J., Antoun, N., Duncan, J. (2005). Attentional functions of parietal and frontal cortex. Cerebral Cortex (New York, N.Y. : 1991), 15(10), 1469-84.

Pisella, L., \& Mattingley, J. B. (2004). The contribution of spatial remapping impairments to unilateral visual neglect. Neuroscience and Biobehavioral Reviews, 28(2), 181-200.

Purcell, B. A., Heitz, R. P., Cohen, J. Y., \& Schall, J. D. (2012). Response variability of frontal eye field neurons modulates with sensory input and saccade preparation but not visual search salience. Journal of Neurophysiology, 108(10), 2737-50.

Rafal, R. D., Posner, M. I., Friedman, J. H., Inhoff, A. W., \& Bernstein, E. (1988). Orienting of visual attention in progressive supranuclear palsy. Brain : A Journal of Neurology, 111 (Pt 2), 267-80.

Rafal, R., \& Posner, M. (1987). Deficits in Human Visual Spatial Attention Following Thalamic Lesions. Proceedings of the National Academy of Sciences of the United States of America, 84(20):7349-53.

Ringman, J. M., Saver, J. L., Woolson, R. F., Clarke, W. R., \& Adams, H. P. (2004). Frequency, risk factors, anatomy, and course of unilateral neglect in an acute stroke cohort. Neurology, 63(3), 468-74.

Rorden, C., Fruhmann Berger, M., \& Karnath, H.-O. (2006). Disturbed line bisection is associated with posterior brain lesions. Brain Research, 1080(1), 17-25.

Rorden, C., Hjaltason, H., Fillmore, P., Fridriksson, J., Kjartansson, O., Magnusdottir, S., \& Karnath, H.-O. (2012). Allocentric neglect strongly associated with egocentric neglect. Neuropsychologia, 50(6), 1151-7.

Saalmann, Y. B., Pinsk, M. a, Wang, L., Li, X., \& Kastner, S. (2012). The pulvinar regulates information transmission between cortical areas based on attention demands. Science (New York, N.Y.), 337(6095), 753-6.

Saj, A., Verdon, V., Vocat, R., \& Vuilleumier, P. (2012). “The anatomy underlying acute versus chronic spatial neglect" also depends on clinical tests. Brain : A Journal of Neurology, 135(Pt 2), e207; author reply e208.

Sapir, A., Soroker, N., Berger, A., \& Henik, A. (1999). Inhibition of return in spatial attention: direct evidence for collicular generation. Nature Neuroscience, 2(12), 1053-4.

Schenkenberg, T., Bradford, D. C., \& Ajax, E. T. (1980). Line bisection and unilateral visual neglect in patients with neurologic impairment. Neurology, 30(5), 509-17.

Schiller, P. H., True, S. D., \& Conway, J. L. (1980). Deficits in eye movements following frontal eye-field and superior colliculus ablations. Journal of Neurophysiology, 44(6), 1175-89.

Shipp, S. (2004). The brain circuitry of attention. Trends in Cognitive Sciences, 8(5), 223-30.

Smith, S. M., Fox, P. T., Miller, K. L., Glahn, D. C., Fox, P. M., Mackay, C. E., Filippini, N., Watkins, K., Toro, R., Laird, A., Beckmann, C. F. (2009). Correspondence of the brain's functional architecture during activation and rest. Proceedings of the National Academy of Sciences of the United States of America, 106(31), 13040-5.

Sprague, J. M. (1966). Interaction of cortex and superior colliculus in mediation of visually guided behavior in the cat. Science (New York, N.Y.), 153(3743), 1544-7.

Thiebaut de Schotten, M., Dell’Acqua, F., Forkel, S. J., Simmons, A., Vergani, F., Murphy, D. G. M., \& Catani, M. (2011). A lateralized brain network for visuospatial attention. Nature Neuroscience, 14(10), 1245-6.

Thiebaut de Schotten, M., Tomaiuolo, F., Aiello, M., Merola, S., Silvetti, M., Lecce, F., Bartolomeo, P., Doricchi, F. (2012). Damage to White Matter Pathways in Subacute and Chronic Spatial Neglect: A Group Study and 2 Single-Case Studies with Complete Virtual "In Vivo" Tractography Dissection. Cerebral Cortex (New York, N.Y. : 1991), 24(3):691-706.

Tournier, J.-D., Calamante, F., \& Connelly, A. (2007). Robust determination of the fibre orientation distribution in diffusion MRI: non-negativity constrained super-resolved spherical deconvolution. Neurolmage, 35(4), 1459-72.

Umarova, R. M., Saur, D., Schnell, S., Kaller, C. P., Vry, M.-S., Glauche, V., Rijntjes, M., Hennig, J., Kiselev, V., 
Weiller, C. (2010). Structural connectivity for visuospatial attention: significance of ventral pathways. Cerebral Cortex (New York, N.Y. : 1991), 20(1), 121-9.

Urbanski, M., Thiebaut de Schotten, M., Rodrigo, S., Catani, M., Oppenheim, C., Touzé, E., Chokron, S.,, Méder, J., Lévy, R., Dubois, B., Bartolomeo, P. (2008). Brain networks of spatial awareness: evidence from diffusion tensor imaging tractography. Journal of Neurology, Neurosurgery, and Psychiatry, 79(5), 598601.

Urbanski, M., Thiebaut de Schotten, M., Rodrigo, S., Oppenheim, C., Touzé, E., Méder, J.-F., Moreau, K., LoeperJeny, C., Dubois, B., Bartolomeo, P. (2011). DTI-MR tractography of white matter damage in stroke patients with neglect. Experimental Brain Research. Experimentelle Hirnforschung. Expérimentation Cérébrale, 208(4), 491-505.

Vaessen, M., Hofman, P., Tijssen, H. N., Aldenkamp, A. P., Jansen, J., \& Backes, W. H. (2010). The effect and reproducibility of different clinical DTI gradient sets on small world brain connectivity measures. Neuroimage, 51(3), 1106.

Vaessen, M., Jansen, J., Braakman, H. M. H., Hofman, P., De Louw, A., Aldenkamp, A. P., \& Backes, W. H. (2014). Functional and structural network impairment in childhood frontal lobe epilepsy. PloS One, 9(3), e90068.

Valero-Cabré, A., Payne, B. R., \& Pascual-Leone, A. (2007). Opposite impact on 14C-2-deoxyglucose brain metabolism following patterns of high and low frequency repetitive transcranial magnetic stimulation in the posterior parietal cortex. Experimental Brain Research, 176(4), 603-15.

Valero-Cabré, A., Payne, B. R., Rushmore, J., Lomber, S. G., \& Pascual-Leone, A. (2005). Impact of repetitive transcranial magnetic stimulation of the parietal cortex on metabolic brain activity: a 14C-2DG tracing study in the cat. Experimental Brain Research, 163(1), 1-12.

Vallar, G. (1998). Spatial hemineglect in humans. Trends in Cognitive Sciences, 2(3), 87-97.

Vallar, G., \& Perani, D. (1986). The anatomy of unilateral neglect after right-hemisphere stroke lesions. A clinical/CT-scan correlation study in man. Neuropsychologia, 24(5), 609-622.

van Assche, M., Kebets, V., Vuilleumier, P., \& Assal, F. (2014). Functional Dissociations Within Posterior Parietal Cortex During Scene Integration and Viewpoint Changes. Cerebral Cortex (New York, N. Y. : 1991).

Verdon, V., Schwartz, S., Lovblad, K.-O., Hauert, C.-A., \& Vuilleumier, P. (2010). Neuroanatomy of hemispatial neglect and its functional components: a study using voxel-based lesion-symptom mapping. Brain : $A$ Journal of Neurology, 133(Pt 3), 880-94.

Vuilleumier, P. (2013). Mapping the functional neuroanatomy of spatial neglect and human parietal lobe functions: progress and challenges. Annals of the New York Academy of Sciences, 1296:50-74.

Vuilleumier, P., Hester, D., Assal, G., \& Regli, F. (1996). Unilateral spatial neglect recovery after sequential strokes. Neurology, 46(1), 184-9.

Vuilleumier, P., Sergent, C., Schwartz, S., Valenza, N., Husain, M., Driver, J., \& Girardi, M. (2007). Impaired perceptual memory of locations across gaze-shifts in patients with unilateral spatial neglect. Journal of Cognitive Neuroscience, 19(8), 1388-406.

Wansard, M., Meulemans, T., Gillet, S., Segovia, F., Bastin, C., Toba, M. N., \& Bartolomeo, P. (2014). Visual neglect: Is there a relationship between impaired spatial working memory and re-cancellation? Experimental Brain Research, 232(10), 3333-43.

Weintraub, S., \& Mesulam, M. (1985). Mental state assessment of young and elderly adults in behavioral neurology. Principles of Behavioral Neurology.

Wilson, B., Cockburn, J., \& Halligan, P. (1987). Development of a behavioral test of visuospatial neglect. Archives of Physical Medicine and Rehabilitation, 68(2), 98-102.

Worsley, K. J., Evans, A. C., Marrett, S., \& Neelin, P. (1992). A three-dimensional statistical analysis for CBF activation studies in human brain. Journal of Cerebral Blood Flow and Metabolism : Official Journal of the International Society of Cerebral Blood Flow and Metabolism, 12(6), 900-18.

zu Eulenburg, P., Caspers, S., Roski, C., \& Eickhoff, S. B. (2012). Meta-analytical definition and functional 
connectivity of the human vestibular cortex. Neurolmage, 60(1), 162-169.

\section{Tables}

\section{Table 1}

\begin{tabular}{lll} 
Perceptual component & $\begin{array}{l}\text { Exploratory visuo-motor } \\
\text { component }\end{array}$ & Allocentric component \\
\hline Line bisection 5 & Schenk ommisions & Clock drawing \\
Line bisection 20 & Bell task ommisions & Scene copying \\
Text reading & Mesulam ommissions & Word transformation \\
\hline
\end{tabular}

Table 1: Behavioral tests included in each of the components. The components and associations with each test were determined by a factorial analysis on all tests given to the patients.

\section{Table2}

\begin{tabular}{|c|c|c|c|c|c|c|c|c|c|c|}
\hline Patient & $\begin{array}{l}\text { Perceptual } \\
\text { component }\end{array}$ & & $\begin{array}{l}\text { Exploratory } \\
\text { visuo- } \\
\text { motor } \\
\text { component }\end{array}$ & & $\begin{array}{l}\text { Allocentric } \\
\text { component }\end{array}$ & & Age & Gender & $\begin{array}{l}\text { Days to } \\
\text { MRI }\end{array}$ & $\begin{array}{l}\text { Days to NP } \\
\text { test }\end{array}$ \\
\hline $\mathrm{p} 1$ & 0.40 & & 0.85 & & 0.16 & & 67 & $M$ & 2 & 10 \\
\hline $\mathrm{p} 2$ & 0.37 & & 1.36 & & 1.24 & & 74 & $\mathrm{~F}$ & 4 & 5 \\
\hline p3 & -0.40 & $*$ & -0.61 & $*$ & -0.80 & $*$ & 61 & $\mathrm{~F}$ & 4 & 11 \\
\hline p4 & -0.25 & $*$ & -0.46 & $*$ & 0.16 & & 78 & $\mathrm{~F}$ & 38 & 34 \\
\hline p5 & -0.26 & $*$ & -0.63 & $*$ & -0.26 & $*$ & 73 & $\mathrm{~F}$ & 1 & 11 \\
\hline p6 & 3.89 & & 2.44 & & 1.24 & & 81 & $\mathrm{~F}$ & 7 & 6 \\
\hline p7 & -0.45 & $*$ & -0.42 & $*$ & -0.80 & $*$ & 52 & $\mathrm{~F}$ & 2 & 18 \\
\hline $\mathrm{p} 8$ & 0.94 & & 2.34 & & 0.70 & & 68 & $M$ & 2 & 14 \\
\hline p9 & -0.37 & $*$ & -0.41 & $*$ & 0.28 & & 65 & $M$ & 8 & 6 \\
\hline
\end{tabular}

Table 2: Component scores, age and gender for each of the included patients. An asterix $\left({ }^{*}\right)$ right of a score indicates this patient was included in the 'good' group for that component. 


\section{Figures legends}

Figure 1. Lesion overlap of all patients. Z-coordinates in MNI range from -12 to 48.

Figure 2: Cluster of WM voxels where damage (FA values) correlated with neglect component scores. (A) Clusters associated with the perceptual component: yellow for cluster 1, blue for cluster 2. (B) Cluster associated with the motor-exploratory component: yellow for cluster one, blue for cluster two. (C) Cluster associated with the allocentric component: yellow for cluster 1, blue for cluster 2, green for cluster 3.

Figure 3. Differences in track density (TD) between the good and bad patient group for the perceptual component. Right side is on the right. Only higher TD was found for the good patient group. Red-yellow colors indicate higher TD for good patients for cluster 1, blue-cyan colors for cluster 2. Parts of the superior longitudinal fasciculus (SLF) in the anterior parietal and posterior temporal regions (top row) and scattered differences in more posterior WM fibers, possibly projecting toward occipital cortex (middle and bottom row). Parts of the cortico-spinal tracts in the anterior capsule, as well as the external capsule (bottom row).

Figure 4: Results for the motor-exploratory component. Same layout and coloring as in Fig. 3. The first and second clusters reveal projections in the superior and middle frontal gyri, near the precentral gyrus, slightly more anterior for the second cluster. Projections in WM near cortical areas presumably corresponding to the frontal eye field (FEF) (bottom row). Projections descending toward the upper midbrain in a region corresponding to the superior colliculus (middle and bottom row).

Figure 5: Results for the allocentric component. Same layout and coloring as in Fig. 3, with the addition of green colors for the third cluster found for this component. Projections in the central fronto-parietal region (more anterior for the second cluster) as well as projections corresponding to the anterior segment of the inferior fronto-occipital fasciculus (IFOF) for the first and third cluster $\left(1^{\text {st }}\right.$ and $2^{\text {nd }}$ row). Projections in the mid-trunk of the corpus callosum and to subcortical structures ( $2^{\text {nd }}$ row). Regions in superior frontal gyrus, more anterior for the second cluster possibly overlapping with the superior frontal occipital fasciculus (SFOF) ( $1^{\text {st }}$ and $2^{\text {nd }}$ row). Midtrunk of the corpus callosum and subcortical structures ( $2^{\text {nd }}$ row). Projections to posterior thalamus and pulvinar for the third cluster (bottom row). 


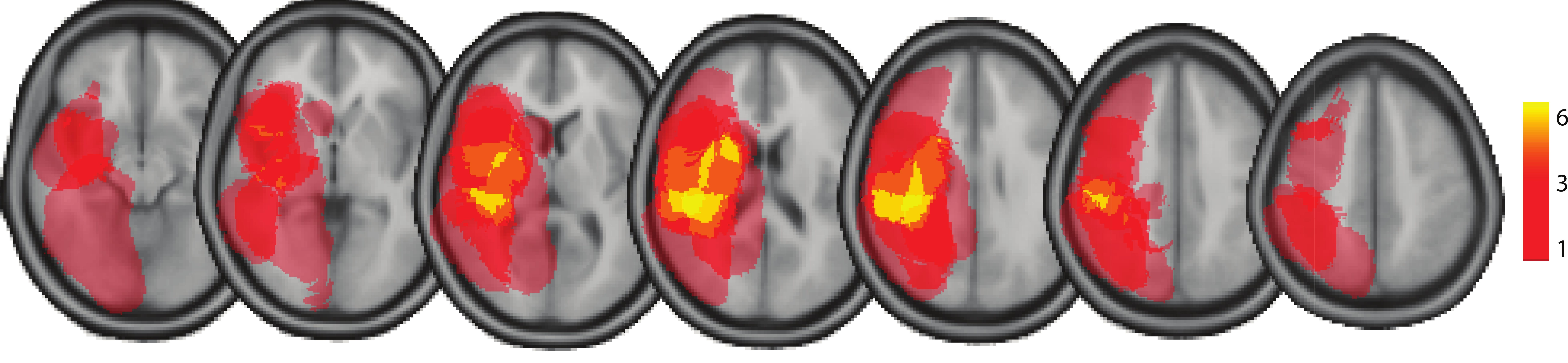




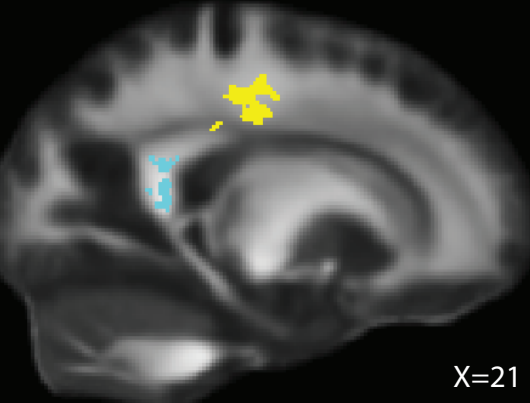

B



R

C

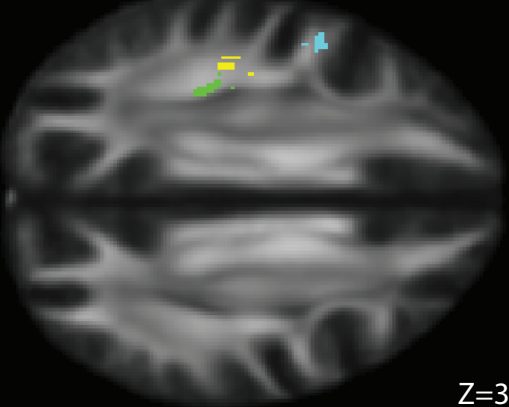

$\mathrm{R}$

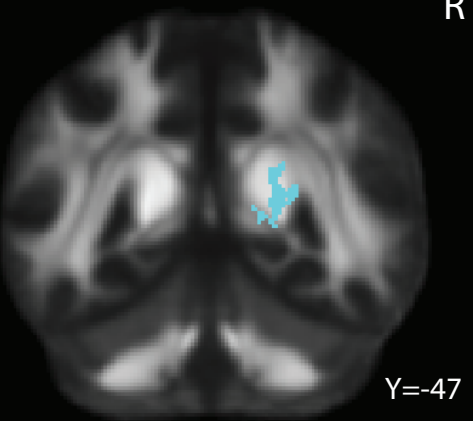

R

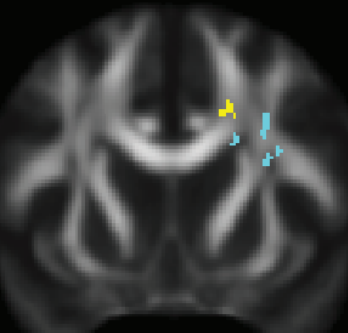

$Y=13$

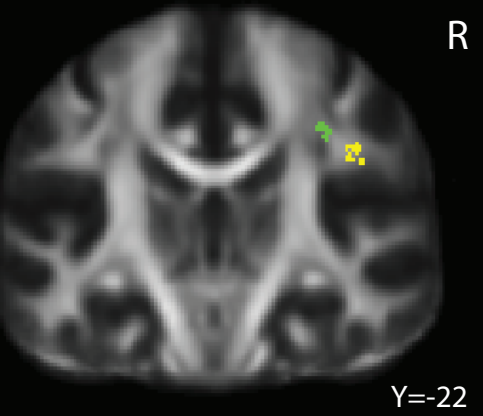



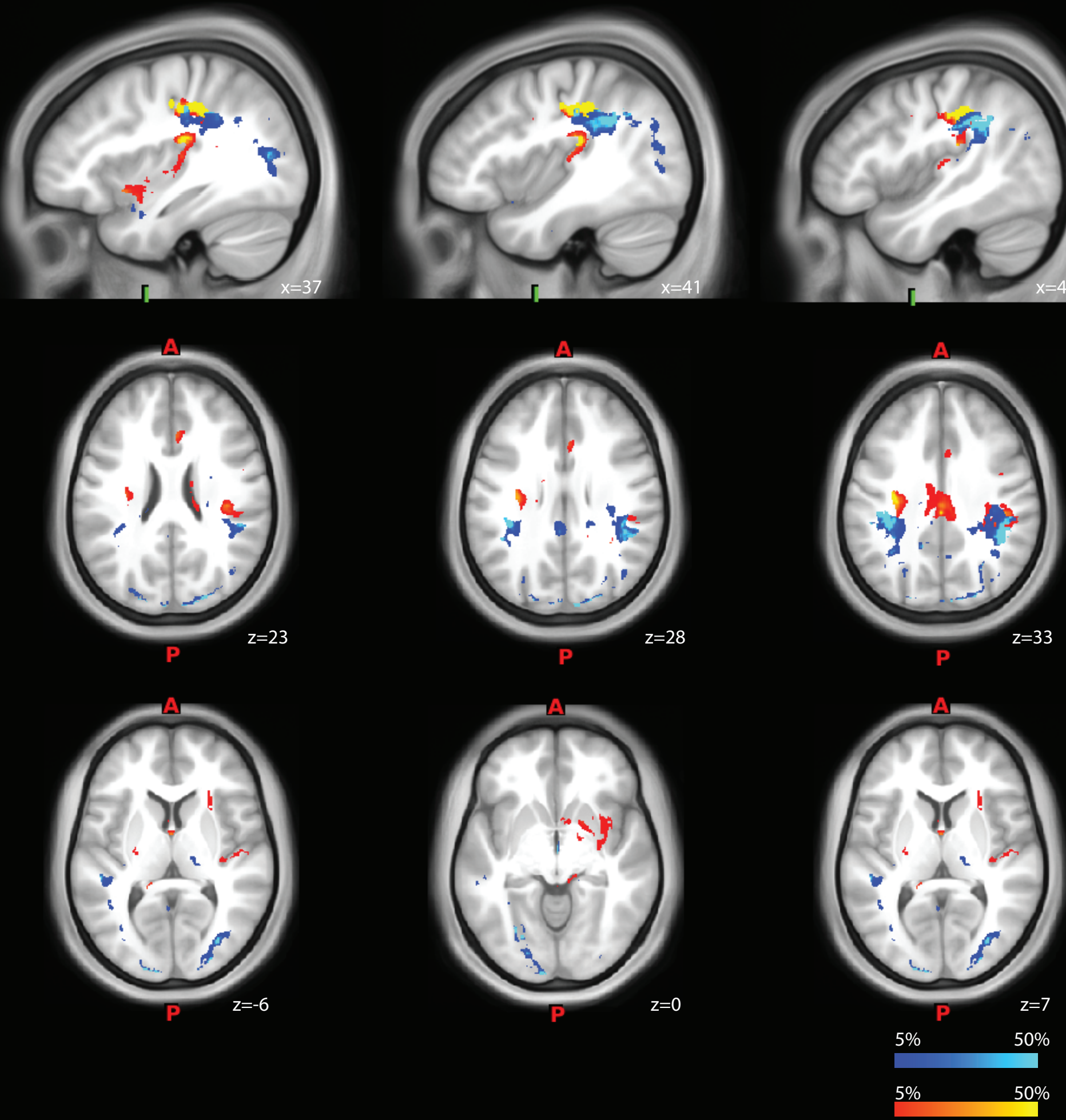
